Supporting information for

\title{
New Method for Atomistic Modeling of the Microstructure of Activated Carbons Using Hybrid Reverse Monte Carlo Simulation
}

\author{
Thanh X. Nguyen ${ }^{\mathrm{a}}$, Nathalie Cohaut ${ }^{\mathrm{b}}$, Jun-Seok Bae ${ }^{\mathrm{a}}$, Suresh K. Bhatia ${ }^{\mathrm{a}}$ * \\ ${ }^{a}$ Division of Chemical Engineering, The University of Queensland, \\ St. Lucia, Brisbane QLD 4067, Australia \\ ${ }^{\mathrm{b}}$ Centre de Recherche sur la Matière Divisée, UMR 6619 1b rue de la férollerie, \\ 45071 Orléans cedex 2, France
}

S1. Derivation of theoretical excess adsorbed quantity used for prediction of experimental adsorption data obtained by the gravimetric technique.

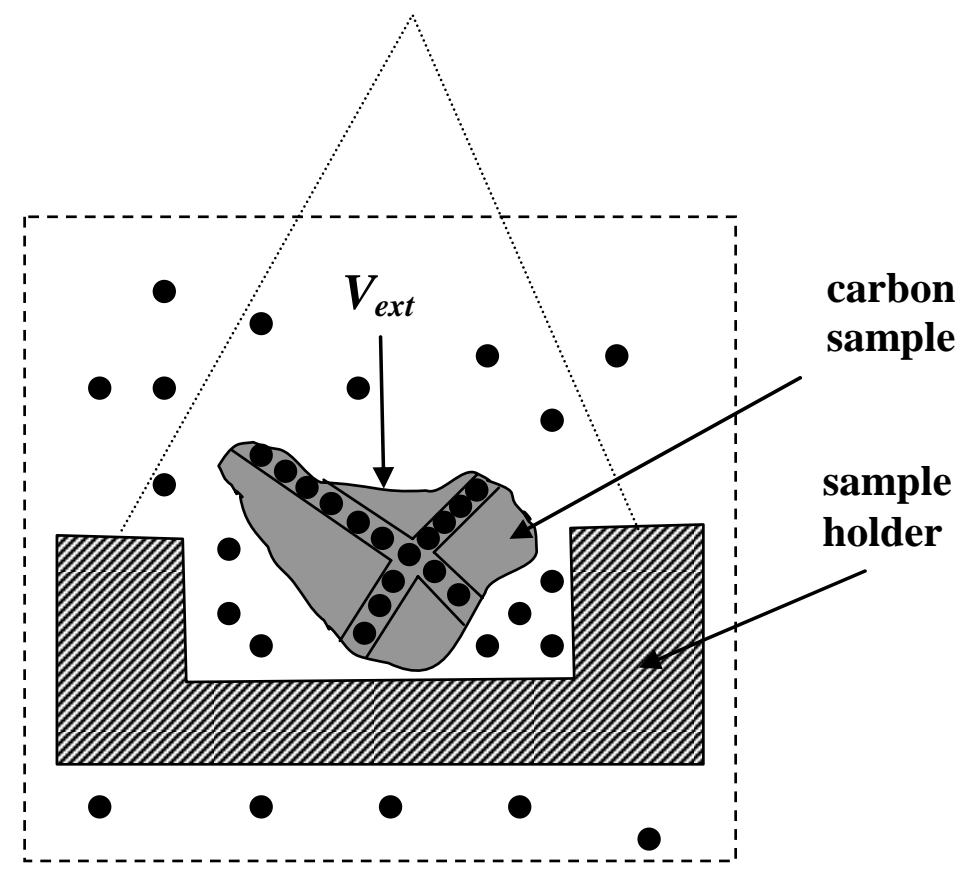

Figure S1. A sketch of gravimetric system used to obtain equilibrium adsorbed quantity. $V_{e x t}$ is the external adsorbent volume of the carbon sample. 
In the gravimetric system sketched in Figure S1, the mass balance is given as

$$
m_{s}^{X}=m_{a}^{X}-\rho_{b}^{X} V_{e x t}^{X}-\rho_{b}^{X} V_{c o n t}^{X}
$$

where $\mathrm{X}$ denotes adsorbing gas. Here $m_{s}^{X}$ and $m_{a}^{X}$ are microbalance signal and absolute adsorbed amount respectively, while $\rho_{b}^{X}$ and $V_{\text {cont }}^{X}$ are bulk density and volume of sample holder respectively. $V_{e x t}^{X}$ is external adsorbent volume defined as the volume of $\mathrm{X}$ in adsorber occupied by the external envelope of the adsorbent particles. Accordingly, the external adsorbent volume comprises of the skeleton and void parts of the adsorbent.

Eq. S1 can be re-written as

$$
\Omega^{X}=m_{s}^{X}+\rho_{b}^{X} V_{\text {cont }}^{X}=m_{a}^{X}-\rho_{b}^{X} V_{e x t}^{X}
$$

where $\Omega^{X}$ is defined as reduced mass.

For the calibration using helium as adsorbing gas, eq. S2 is re-written in the form

$$
\Omega^{H e}=-\left(V_{e x t}^{H e}-\frac{m_{a}^{H e}}{\rho_{b}^{H e}}\right) \rho_{b}^{H e}
$$

At sufficiently high temperature, $\frac{m_{a}^{H e}}{\rho_{b}^{H e}}$ is almost constant, and the reduced mass, $\Omega^{H e}$, is therefore linearly dependent upon bulk density of helium. Accordingly, the expression, $V_{e x t}^{H e}-\frac{m_{a}^{H e}}{\rho_{b}^{H e}}$, is the effective helium adsorbent volume, $V_{a d s}^{H e}$, routinely used to determine experimental adsorbed amount by the gravimetric technique. In other words, the effective helium adsorbent volume is given as 


$$
V_{a d s}^{H e}=V_{e x t}^{H e}-\frac{m_{a}^{H e}}{\rho_{b}^{H e}}
$$

Also, from eq. S2, the mass balance for an adsorbing gas $(f)$ different from helium is written as

$$
\Omega^{f}=m_{a}^{f}-V_{e x t}^{f} \rho_{b}^{f}
$$

If deformation of the porous sample is taken into account, the external adsorbent volume in the fluid $(f), V_{e x t}^{f}$, is given as

$$
V_{e x t}^{f}=V_{e x t}^{H e}+\Delta V
$$

where $\Delta V$ is the change in the external adsorbent volume of porous carbon in the gas $(f)$ from that in helium. If the external helium adsorbent volume is considered as reference adsorbent volume, $\Delta V$ is excess adsorbent volume. Accordingly, positive $\Delta V$ indicates swelling and negative $\Delta V$ indicates shrinkage.

Upon substitution of eqs.S4 and S6 into eq.S5, we obtain an explicit expression for the excess adsorbed quantity in a non-rigid porous carbon as follows:

$$
\Omega^{f}+V_{a d s}^{H e} \rho_{b}^{f}=m_{a}^{f}-\frac{m_{a}^{H e}}{\rho_{b}^{H e}} \rho_{b}^{f}-\Delta V \rho_{b}^{f}
$$

It can now be clearly seen that the L.H.S. of eq.S7 is the experimental adsorption quantity obtained by the gravimetric technique. Accordingly, if the R.H.S. of eq.S7 is defined as the theoretical excess adsorbed quantity, the consistency between theoretical excess adsorbed quantity and its experimental counterpart is achieved. As a result, theoretical excess adsorbed quantity of adsorbing gas $(f), m_{e x}^{f}$, is now defined as 


$$
m_{e x}^{f}=m_{a}^{f}-\frac{m_{a}^{H e}}{\rho_{b}^{H e}} \rho_{b}^{f}-\Delta V \rho_{b}^{f}
$$

The excess adsorbent volume, $\Delta V$, in eq.S8 is unknown, and varies with adsorptive pressure. However, for a somewhat rigid activated carbon it can be deduced that the excess adsorbent volume, $\Delta V$, may vary significantly on the left adsorption branch due to strong adsorption in this region, while varying very slowly on the right adsorption branch due to the fact that interlayer spacing in the adsorbent reduces to an equilibrium value due to the compression on the left adsorption branch, leading to mechanical stability of the adsorbent on the right adsorption branch. On the other hand, the bulk density of adsorbing gas, $\rho_{b}^{f}$, is normally small on the left adsorption branch in comparison with adsorbed density, leading to negligible contribution of the last term in the R.H.S. of eq.S8. As a consequence, the excess adsorbent volume in eq. S8 can be assigned to its largest value, $\Delta V_{\max }$, in the investigated pressure range. For sake of clarity, eq. $\mathrm{S} 8$ can be rewritten as

$$
m_{e x}^{f}=m_{a}^{f}-\frac{m_{a}^{H e}}{\rho_{b}^{H e}} \rho_{b}^{f}-\Delta V_{\max } \rho_{b}^{f}
$$

